\title{
Excitation of Coherent Oscillations in Underdoped Cuprate Superconductors by Intense THz Pulses
}

\author{
Matthias C. Hoffmann*a, Wei-Sheng Lee ${ }^{a}$, Georgi L. Dakovski ${ }^{\text {a }}$, Joshua J. Turner ${ }^{\text {a }}$, Simon M. \\ Gerber $^{\mathrm{b}}$, Doug Bonn ${ }^{\mathrm{c}}$, Walter Hardy ${ }^{\mathrm{c}}$, Ruixing Liang ${ }^{\mathrm{c}}$, Marco Salluzzo ${ }^{\mathrm{d}}$ \\ a SLAC National Accelerator Laboratory, 2575 Sand Hill Road, Menlo Park, CA, USA 94025, \\ ${ }^{b}$ Paul Scherrer Institut (Switzerland) \\ ${ }^{\mathrm{c}}$ Department of Physics and Astronomy, University of British Columbia, 6224 Agricultural Rd, \\ Vancouver, BC V6T 1Z1 \\ ${ }^{\mathrm{d}}$ CNR-INFM and Dipartimento di Scienze Fisiche, Università di Napoli "Federico II" Piazzale \\ Tecchio 80, I-80125 Napoli, Italy
}

\begin{abstract}
We use intense broadband $\mathrm{THz}$ pulses to excite the cuprate superconductors $\mathrm{YBCO}$ and $\mathrm{NBCO}$ in their underdoped phase, where superconducting and charge density wave ground states compete. We observe pronounced coherent oscillations at attributed to renormalized low-energy phonon modes. These oscillation features are much more prominent than those observed in all-optical pump-probe measurements, suggesting a different excitation mechanism.
\end{abstract}

Keywords: Nonlinear THz, high-temperature superconductivity, charge density wave

\section{INTRODUCTION}

In order to gain insight into the mechanism of high-temperature superconductivity in the cuprate materials family, understanding of the spin, charge and orbital correlations in both the normal and superconducting states is necessary. Well-known examples are the antiferromagnetic ordering in the undoped, insulating parent compounds and the stripe order at 1/8 doping in $\mathrm{La}_{2-\mathrm{x}} \mathrm{Sr}_{\mathrm{x}} \mathrm{CuO}_{4}$ and related systems [1,2]. Renewed interest has been focused on the $\mathrm{RBa}_{2} \mathrm{Cu}_{3} \mathrm{O}_{6+\mathrm{x}}$ family (where $R=\mathrm{Y}$ or another rare earth element), which possess much higher critical temperature $\left(\mathrm{T}_{\mathrm{c}}\right)$ and the show perplexing features such as the "pseudogap" region of the phase diagram.

A recent development is the discovery of charge density wave (CDW) order in the $R \mathrm{Ba}_{2} \mathrm{Cu}_{3} \mathrm{O}_{6+\mathrm{x}}$ system using resonant soft X-ray scattering [3] and hard-X-ray scattering methods [4]. The charge density wave amplitude was shown to initially increase with lower temperature but to subsequently decline for temperatures below Tc, hinting at competition between the superconducting and charge density order.

Dynamics related to the charge density have been observed with ultrafast laser spectroscopy methods in the time domain $[5,6]$. Specifically, Hinton et al observed $1.8 \mathrm{THz}$ oscillations in a bulk sample of underdoped $\mathrm{YBa}_{2} \mathrm{Cu}_{3} \mathrm{O}_{6+x}(\mathrm{YBCO})$ at $x=0.67$. Their results are based on pump-probe experiments with femtosecond laser pulses at $800 \mathrm{~nm}$, corresponding to photon energy of $1.55 \mathrm{eV}$, and excitation densities of $1.5 \mu \mathrm{J} / \mathrm{cm}^{2}$. Under these conditions, the oscillatory component of the pump probe signal is superimposed on an incoherent and exponentially decaying background that is about 1 order of magnitude larger. This incoherent background is due to the fact that the excitation photon energy is above the band gap and many excitation pathways are accessible. In this work, extending our results of [7], we investigate excitation of underdoped YBCO and NBCO samples by an electromagnetic field pulse with frequency content on the energy scale of the CDW order. The photon energy of these single-cycle THz pulses is in the range of 1 to $10 \mathrm{meV}$ and well below any band gap energy or known optical phonon frequency. Simultaneously, the electrical field strength and the intensity of the $\mathrm{THz}$ pulses is large enough to trigger a coherent response of the sample.

*hoffmann@slac.stanford.edu;

phone

$1-650-926-4446$

www.slac.stanford.edu

Ultrafast Bandgap Photonics, edited by Michael K. Rafailov, 


\section{EXPERIMENTAL}

\subsection{THz pump - near infrared probe apparatus}

Single-cycle THz pulses with $4 \mu \mathrm{J}$ energy and peak fields up to $400 \mathrm{kV} / \mathrm{cm}$ were generated by optical rectification in $\mathrm{LiNbO}_{3}$ using the tilted pulse front method [8,9]. The $\mathrm{THz}$ beam was focused onto the sample located inside a closedcycle helium cryostat using off-axis parabolic mirrors. The maximum pump fluence was on the order of $0.2 \mathrm{~mJ} / \mathrm{cm}^{2}$ and could be attenuated continuously using a combination of two wire grid polarizers. The repetition rate of the laser system was $1 \mathrm{kHz}$. Weak probe pulses at $800 \mathrm{~nm}$ wavelength and $100 \mathrm{fs}$ duration were reflected off of the sample and detected with a photo-diode (Fig 1). The pump pulses were modulated at $500 \mathrm{~Hz}$ and a lock-in amplifier was used to acquire the change in sample reflectivity. The sample reflectivity at $800 \mathrm{~nm}$ is a proxy for overall conductivity since the photon energy is above any free-carrier Drude resonance.

The shape THz field at the position of the sample was characterized in he time domain using a 100 um thick $<110>\mathrm{GaP}$ crystal on a $<100>$ substrate mounted in the sample plane. The peak $\mathrm{THz}$ field strength can be inferred directly from the polarization modulation of the transmitted probe pulses caused by the electro-optic effect.

The orientation of the sample relative to the $\mathrm{THz}$ polarization as well as the direction of the probe polarization with respect to the $\mathrm{THz}$ polarization was changed for different set of data.

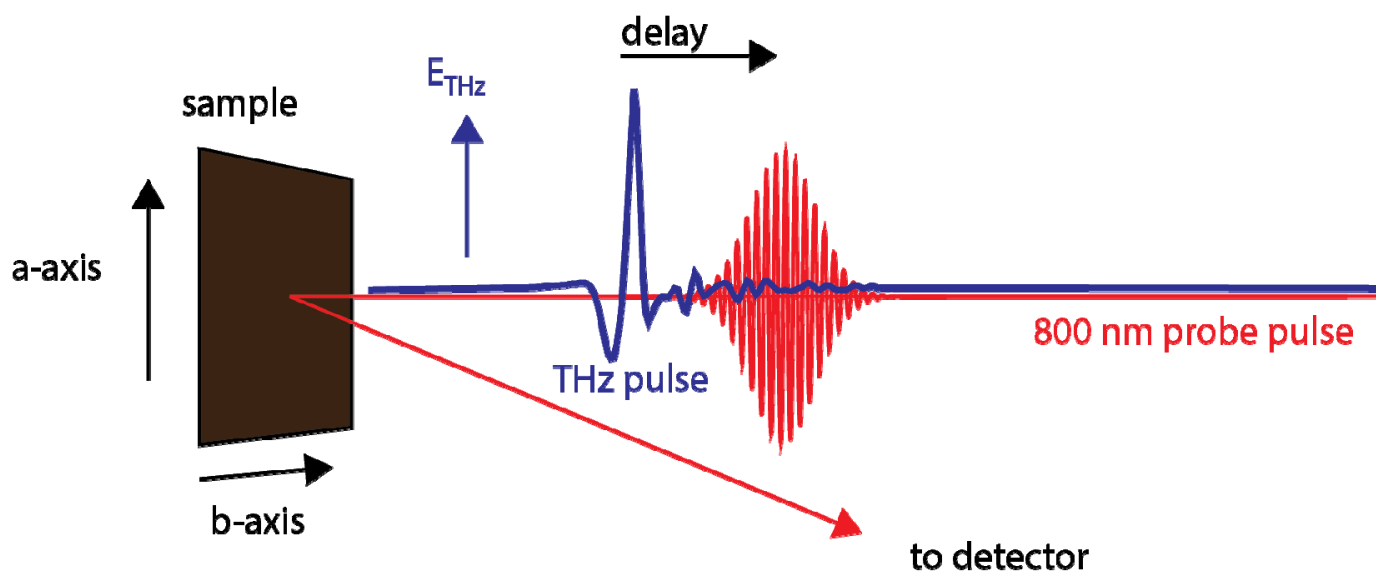

Figure 1: Experimental geometry: single-cycle THz pulses with energy up to $4 \mathrm{uJ}$ are incident on the sample mounted inside a cryostat. Weak $800 \mathrm{~nm}$ probe pulses with $100 \mathrm{fs}$ duration are incident collinearly and reflected off the sample which is kept at a slight angle. The orientation of the sample and the probe polarization was varied with respect to the $\mathrm{THz}$ polarization.

\subsection{Samples}

We studied a number of different samples from the "123" family of cuprate superconductors. The first sample was a $\mathrm{YBa}_{2} \mathrm{Cu}_{3} \mathrm{O}_{6.75}$ ortho-III single crystal with $\mathrm{x}=0.75$, hole doping $\mathrm{p}=0.13$, and $\mathrm{T}_{\mathrm{c}}$ of $75.2 \mathrm{~K}[10,11]$ (UD-YBCO sample). The second sample was a optimally doped YBCO orthorhombic (twinned) thin film on $\mathrm{LaAlO}_{3}$ substrate. The film thickness was $\sim 200 \mathrm{~nm}$ and $\mathrm{Tc} \sim 90 \mathrm{~K}$ [3]. The last sample was underdoped $\mathrm{Nd}_{1.2} \mathrm{Ba}_{1.8} \mathrm{Cu}_{3} \mathrm{O}_{7}$ tetragonal (untwinned) thin film on $\mathrm{SrTiO}_{3}$. The thickness was $\sim 100 \mathrm{~nm}$ with the critical temperature $\mathrm{Tc} \sim 65 \mathrm{~K}$, and the charge order temperature $\mathrm{T}_{\mathrm{CDW}} \sim 150 \mathrm{~K}[12]$. 


\section{RESULTS}

\subsection{Single crystalline UD-YBCO}

Figure 2a shows the THz-induced pump-probe signal at 10K for both pump and probe fields parallel to the sample baxis. We observe an initial peak that tracks the square of the THz field. This is followed by coherent oscillations with a period of $540 \mathrm{fs}$. The maximum reflectivity change $\Delta \mathrm{R} / \mathrm{R}$ is about to $10^{-3}$, which is about two times higher than what was reported in Ref [6]. The oscillatory part of the signal is much larger than any incoherent background at this temperature.
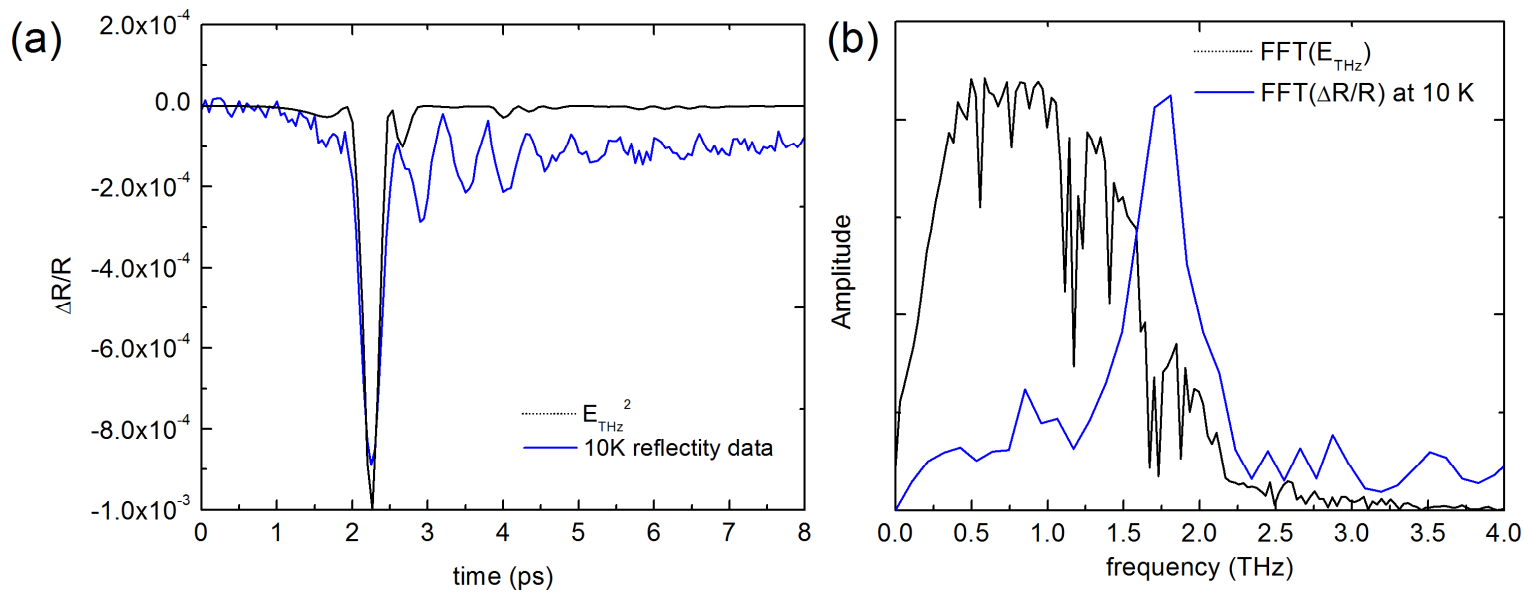

Figure 2: (a) THz-induced reflectivity change in single crystal $\mathrm{YBa}_{2} \mathrm{Cu}_{3} \mathrm{O}_{6.75}$ at $10 \mathrm{~K}$ for $\mathrm{E}_{\mathrm{THz}}$ and $\mathrm{E}_{\text {probe }}$ both parallel to the crystallographic b-axis. The sign of the signal changes above Tc. ETHz2 is shown for reference. (b) FFT amplitude spectra of the $10 \mathrm{~K}$ data for delay $>2.5 \mathrm{ps}$ and after background subtraction. The dashed line is the amplitude spectrum of the pump THz pulse.

The frequency spectrum of the pump probe signal for $\mathrm{t}>2.5 \mathrm{ps}$ is shown in Fig $2 \mathrm{~b}$. A strong mode at $1.85 \mathrm{THz}$ is clearly visible. The full-width half maximum is $0.5 \mathrm{THz}$ corresponding to a lifetime of about $2 \mathrm{ps}$. We note that the sign of the pump-probe signal is inverted if we change the probe laser pulse polarization from the b-axis to the a-axis.

Figure 3 shows the temperature dependence of the THz induced reflectivity change. While the $1.85 \mathrm{THz}$ oscillations are clearly pronounced at low temperature, they are getting damped with increasing temperature and disappear around 55 $\mathrm{K}$, well below $\mathrm{T}_{\mathrm{c}}=75 \mathrm{~K}$. Simultaneously, we observe the appearance slow relaxation dynamics in the form of an exponential decay. The time constant of this decay and the total amplitude of the signal increase dramatically when approaching the critical temperature.

We still observe a pump probe signal above Tc that persists up to approximately $200 \mathrm{~K}$, but it is much weaker and largely featureless. It shows no oscillatory components and can be described by an exponential decay convoluted with the $\mathrm{THz}$ pump shape. If the probe polarization is along the b-axis, the sign of the reflectivity signal changes when crossing Tc. The period and hence the central frequency of the observed oscillation mode does not change appreciably with temperature. Figure $3 \mathrm{~b}$ shows damped sine fits to the oscillatory components of he pump probe signal (after background subtraction) for several sample temperature. Contrary to Ref [6] we do not observe a phase shift when approaching Tc from low temperatures before the oscillations disappear entirely. 

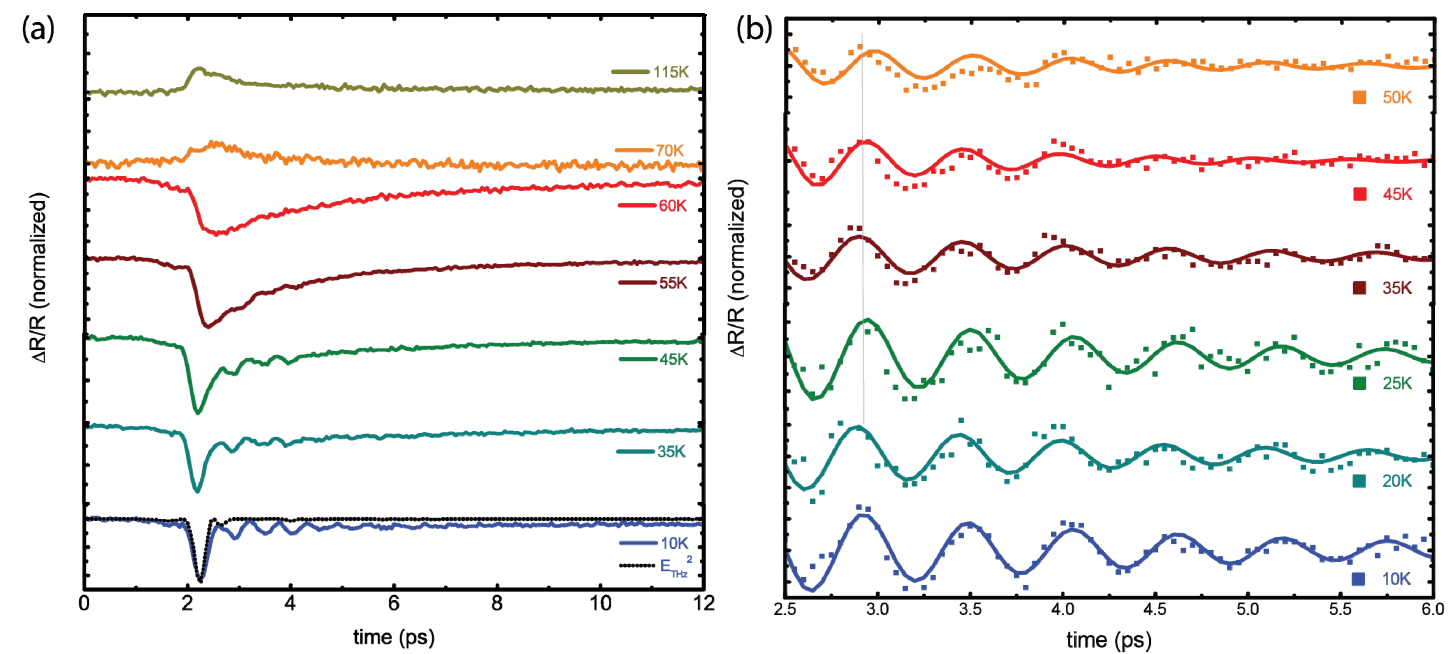

Figure 3: (a) Normalized THz-induced reflectivity change in single-crystal UD-YBCO at various temperatures. THz pump polarized along b axis, $800 \mathrm{~nm}$ probe along b-axis. $\mathrm{E}_{\mathrm{THz}}^{2}$ is shown for reference in the $10 \mathrm{~K}$ data (b) Coherent oscillations in the time domain at different temperatures. Solid line is a damped sine fit after background subtraction. We do not observe a $\pi / 2$ phase shift in the signal when approaching Tc.

The dependence of the dynamical reflectivity change on the THz field strength, was studied via controlled attenuation of the THz pump beam by a pair of THz linear polarizers. Figure 4 shows the scaling of the peak pump-probe signal with the $\mathrm{THz}$ field strength at $8 \mathrm{~K}$ and $55 \mathrm{~K}$. In both cases the dependence is quadratic.
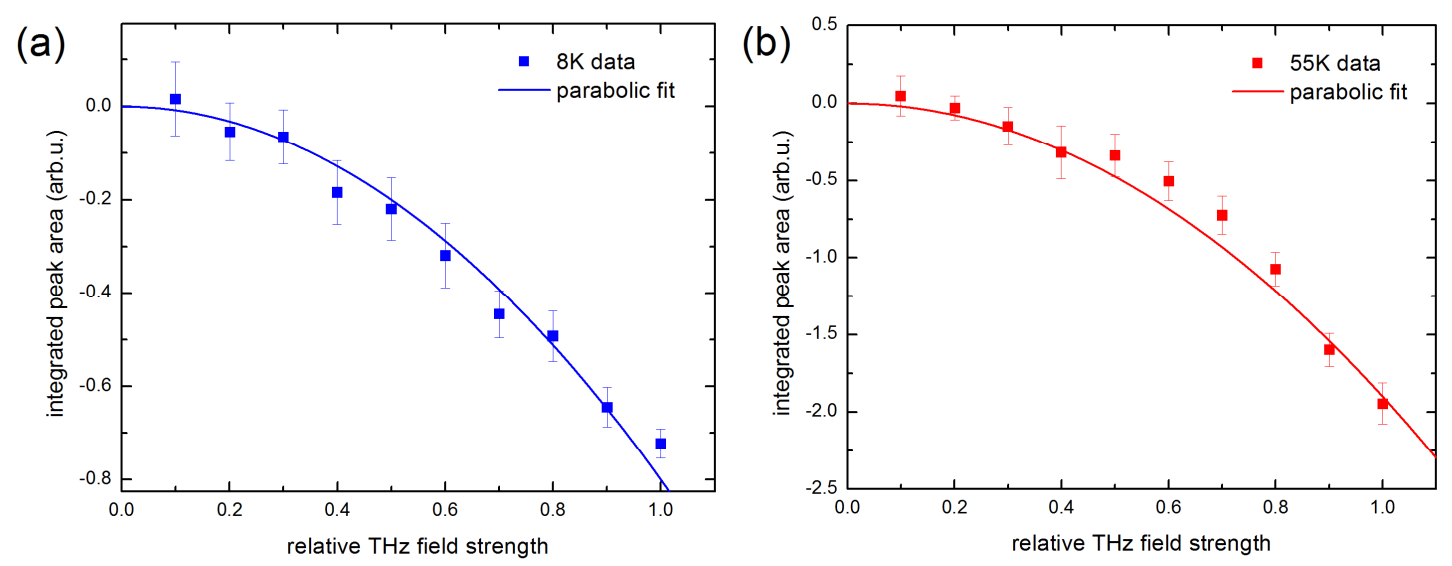

Figure 4: THz field dependence of the time resolved reflectivity signals at (a) $8 \mathrm{~K}$ and (b) $55 \mathrm{~K}$ for ETHz||Eprobe||b-axis. The $\mathrm{THz}$ field was attenuated with a pair of wiregrid polarizers.

When the polarization of the THz pump beam is along the sample a-axis, we observe a qualitatively similar signal but with the opposite sign of $\Delta \mathrm{R} / \mathrm{R}$. The Fourier transformation of the oscillatory signal reveals the presence of an additional mode centered at $2.65 \mathrm{THz}$. This higher frequency mode matches the energy shift recently found in observations of phonon anomalies coupled to the CDW mode [13]. 

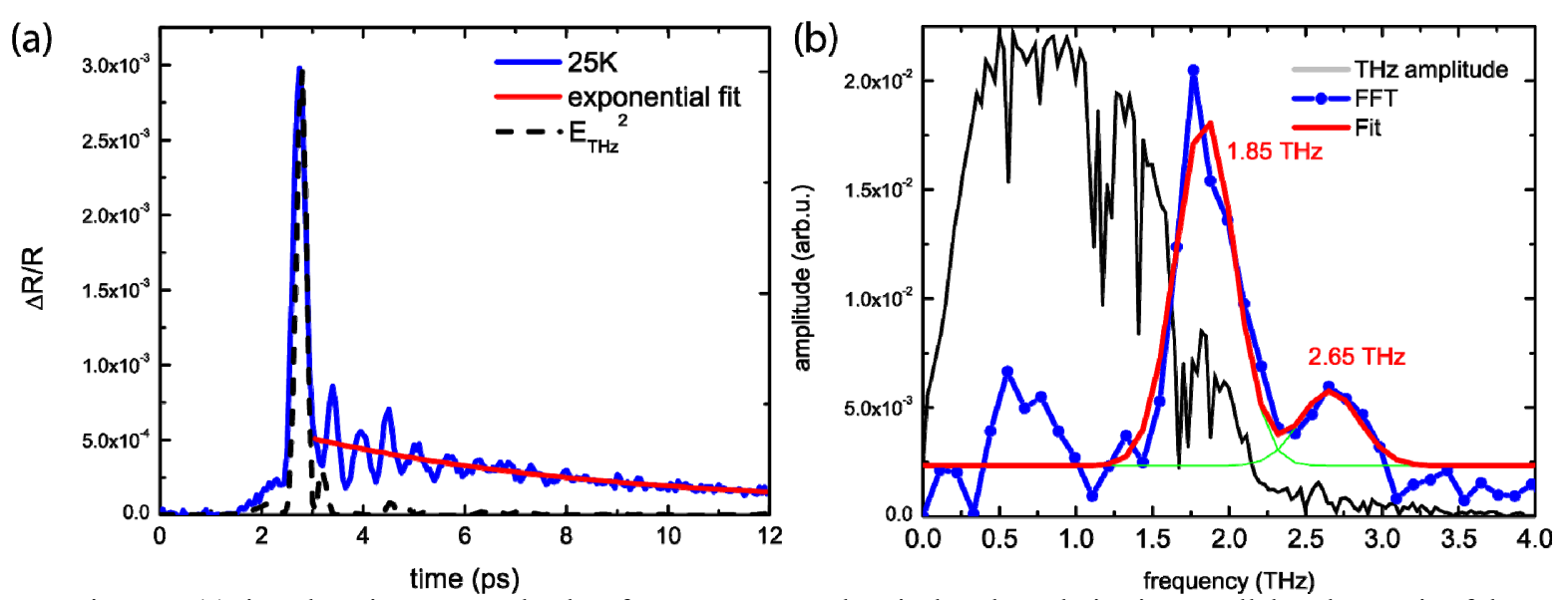

Figure 5: (a) time domain pump probe data for THz pump and optical probe polarization parallel to the a-axis of the sample (blue curve). The oscialltory part of the signal is superimposed on an incoherent background that can be described by a single-exponential decay (red curve) (b) amplitude spectrum of the oscillatory part of the signal (blue curve) and Gaussian fits (red curve). The THz amplitude spectrum is shown for reference (black)

\subsection{Optimally doped YBCO thin film}

In addition to the underdoped single crystalline YBCO sample, we also carried out a series of measurements on an optimally doped YBCO thin film on LaAlO3 substrate. The film thickness was $200 \mathrm{~nm}$ and the superconducting transition temperature Tc $\sim 90 \mathrm{~K}$. Here, the THz-induced reflectivity change was markedly different form the underdoped case (Figure 6). The sample response follows the square of the THz pulse, but no oscillatory component is visible at any temperature or probe condition. Above $\mathrm{T}_{\mathrm{c}}$, the signal decreases and a slow rising component followed by long-lived offset is observed after the $\mathrm{THz}$ excitation.
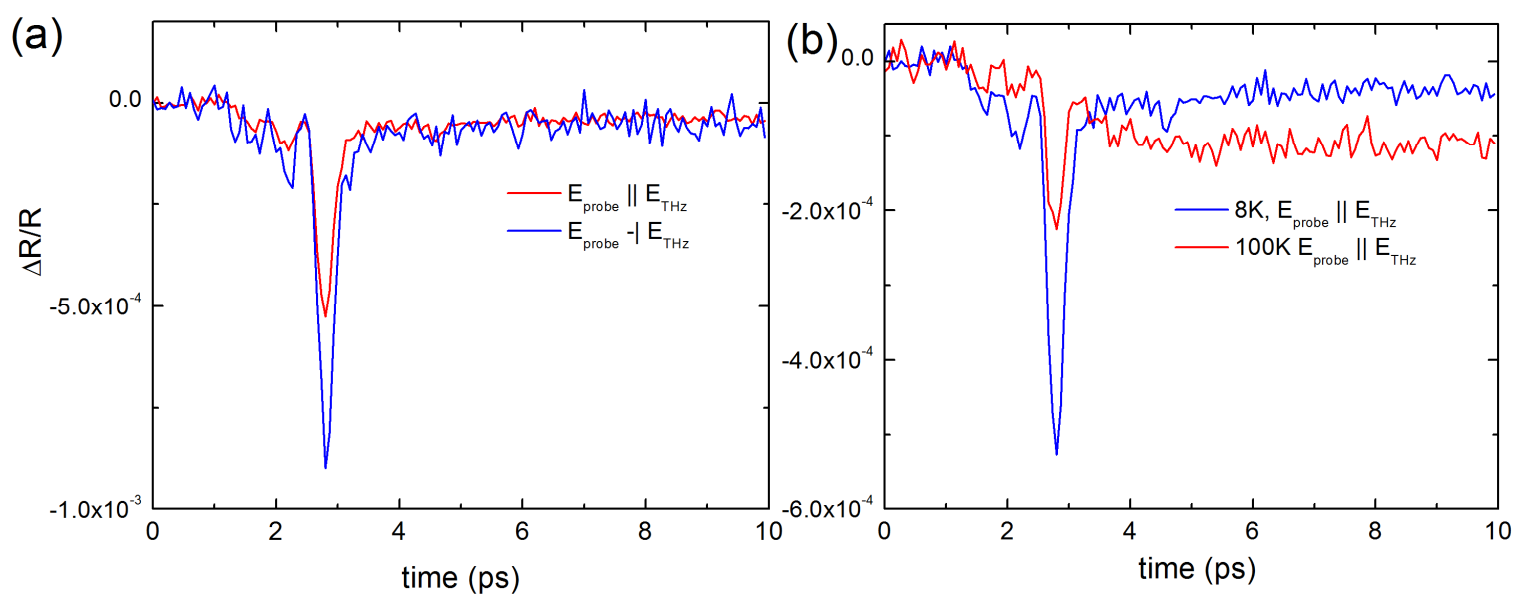

Figure 6: (a) THz pump/800 nm probe signal from optimally doped YBCO on LAO at $8 \mathrm{~K}$ for two different probe polarizations. The signals are compatible with the envelope of the pump pulse and show no other features.(b) signal at $8 \mathrm{~K}$ and above $\mathrm{T}_{\mathrm{c}}$ at $100 \mathrm{~K}$.

\subsection{Results for UD-NBCO}

In order to gain insight into the dynamics of charge density wave order in a closely related materials system, we carried out a series of measurements on underdoped $\mathrm{Nd}_{1.2} \mathrm{Ba}_{1.8} \mathrm{Cu}_{3} \mathrm{O}_{7}$ (UD-NBCO). The sample was a 100 nm thick untwinned thin film on a SrTiO3 substrate with $\mathrm{T}_{\mathrm{c}} \sim 65 \mathrm{~K}$, and the charge order temperature $\mathrm{T}_{\mathrm{CDW}} \sim 150 \mathrm{~K}$ [12]. The results are summarized in Figure 7. 
At low temperature, we observe an initial peak with a rising edge shape that tracks the square of the THz field. The peak reflectivity change $\Delta \mathrm{R} / \mathrm{R}$ is on the order of $10^{-3}$, which is similar to the values in our previous experiment on YBCO [7] and about $2 \mathrm{x}$ higher than reported in [6].

This is followed by coherent oscillations corresponding to frequencies at $1.5 \mathrm{THz}$ and possibly $0.45 \mathrm{THz}$, superimposed on an exponential decay (Figure $7 \mathrm{~b}$ ). The main oscillation mode has and a full width half maximum corresponding to a lifetime of about 2 ps.
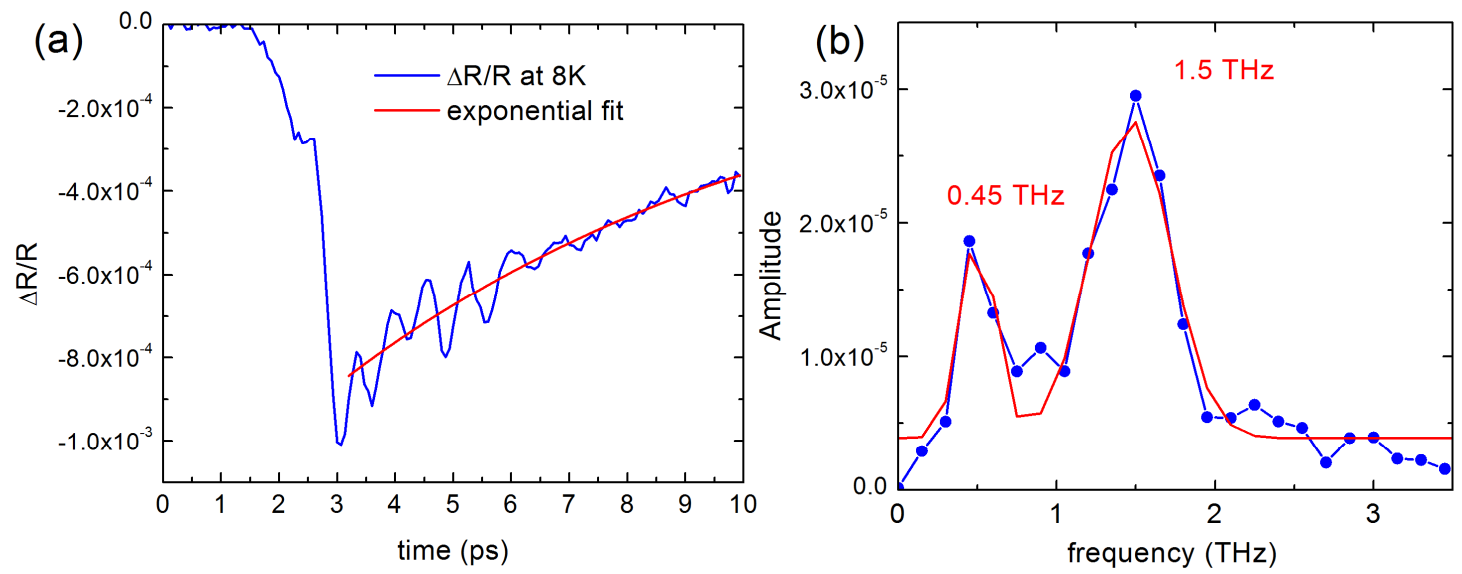

Figure 7: (a) UD-NBCO reflectivity change for THz-pump/800 nm probe at $8 \mathrm{~K}$ and (b) spectral components of the observed coherent oscillations after subtraction of the exponentially decaying background.

Figure 8 shows the temperature dependence of the THz-induced reflectivity change. The absolute strength of the signal decreased with increasing temperature, but a small signal persists above $T_{c}$. The oscillatory part of the signal gets rapidly damped when approaching Tc and disappears entirely around $50 \mathrm{~K}$. The central frequency of the observed oscillation mode does not change appreciably with temperature. We note that the exponentially decaying part of the signal gets faster with higher temperature, contrary to the UD-YBCO measurements where we saw the slowest dynamics just below Tc. The relationship between temperature and the time constant of the incoherent decay from single- exponential fits is shown in Figure 8b.
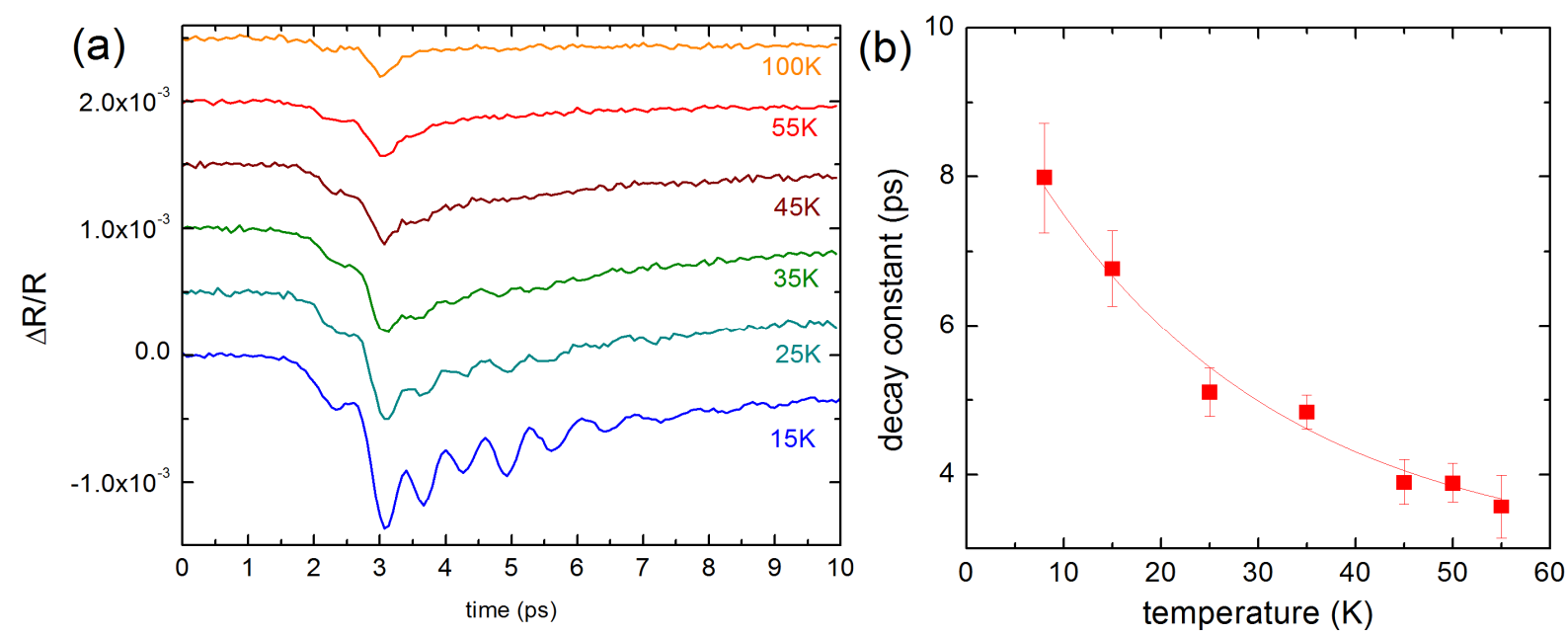

Figure 8 Temperature dependence of the THz pump/optical probe signal in underdoped NBCO. The oscillatory feature disappears in the vicinity of Tc. (b) Decay constants from a single-exponential fit to the signal for time delays $>3$ ps. The solid line is a guide to the eye. 


\section{DISCUSSION}

The coherent oscillations observed by transient reflectivity in both underdoped systems are likely related to their charge density wave ordered state. In UD-YBCO, the $1.85 \mathrm{THz}$ and $2.65 \mathrm{THz}$ modes match the energies of the strongly renormalized phonon modes at the wave vector corresponding to the CDW modulation, measured by inelastic x-ray scattering [13]. While these softened modes are located at a finite momentum, they are still optically accessible at the zone center due to Brillouin zone folding with the CDW breaking the translational symmetry. While CDW-related oscillations have been observed in optical pump experiments on YBCO and LSCO before [6,5], strong-field THz pulses prove to be a surprisingly effective way to trigger this effect. Since the photon energy of the THz pulses in in the range of $\mathrm{meV}$, direct creation of Cooper pair breaking and quasiparticle generation is not possible. Instead, the excitation mechanism must be driven by the electric field component of the THz pulse. Given the considerable field strength, the local current may approach the de-pairing current density of $3 \times 10^{12} \mathrm{~A} / \mathrm{m}^{2}$ in $\mathrm{YBCO}$ [14].

We note that the temperature dependent dynamics are found to be very different than in previous optical pump experiments. First, while the collective oscillations induced by optical excitation persist to temperatures well above Tc, and are detectable up to $\mathrm{T}_{\text {cdw }}$, the THz-induced collective dynamics disappear slightly below Tc (Figure 3a). Secondly, unlike optical excitations, neither the frequency nor the phase of the oscillations shift appreciably as a function of temperature. Lastly, the temperature dependence of the incoherent dynamics is also very different. In UD-YBCO the

| temperature evolution of the recovery time displays an increase just below Tc, a behavior often ascribed to the presence of a "phonon bottleneck" where photoexcited quasiparticles and low-energy phonons are in a state of quasi-equilibrium [15]. On the other hand, the amplitude of incoherent background signal vanishes at low temperatures, in contrast with the temperature-independent behavior observed in all-optical experiments. This may be due to the increase in de-pairing current density with lower temperature.

In UD-NBCO the temperature dependent dynamics show astonishingly different behavior. At low temperature an oscillation mode at $1.5 \mathrm{THz}$ superimposed on an exponentially decaying background is clearly visible, with a possible side band at $0.45 \mathrm{THz}$. However, the decay constant of the incoherent portion of the signal decreases with higher temperature, in sharp contrast to the observations in UD-YBCO.

\section{CONCLUSION}

We demonstrate that intense broadband THz pulses can be used to induce strong coherent oscillations at low temperatures in underdoped YBCO and NBCO. These can be attributed to the strong interaction between the superconducting state and the CDW mode. The excitation mechanism is likely based on the instantaneous current driven by the THz field in contrast to direct photon absorption in all optical spectroscopy methods. This unique excitation pathway will enable future investigations that may provide more information by directly monitoring the $\mathrm{CDW}$ dynamics with advanced probe methods like ultra-fast resonant $\mathrm{x}$-ray scattering. These could reveal the interplay between density wave physics and SC, thought to be of importance in all families of cuprates, and can be used as a novel way of exploring the properties of high-temperature superconductors.

\section{ACKNOWLEDGEMENTS}

Portions of this research were carried out at the Linac Coherent Light Source (LCLS) at the SLAC National Accelerator Laboratory. LCLS is an Office of Science User Facility operated for the U.S. Department of Energy Office of Science by Stanford University.

\section{REFERENCES}

[1] Tranquada, J. M. , Sterniel, B.J., Axe, J.D., Nakamura, Y., Uchida, S., "Evidence for stripe correlations of spins and holes in copper oxide superconductors," Nature 375, 561 (1995)

[2] Abbamonte, P., Rusydi, A., Smadici, S., Gu, G. D., Sawatzky, G. A., Feng, D. L., "Spatially modulated 'Mottness' in $\mathrm{La}_{2-x} \mathrm{Ba}_{x} \mathrm{CuO}$," Nat. Phys. 1, 155 (2005) 
[3] Ghiringhelli, G. et al., "Long-range incommensurate charge fluctuations in (Y, Nd)Ba2Cu3O6+x. ," Science, 337, 821 (2012).

[4] Chang, J. et al "Direct observation of competition between superconductivity and charge density wave order in $\mathrm{YBa}_{2} \mathrm{Cu}_{3} \mathrm{O}_{6.67}$," Nature Phys. 8, 871 (2012).

[5] Torchinsky, D.H., Mahmood, F., Bollinger, A.T. Božović, I., and Gedik, N. "Fluctuating chargedensity waves in a cuprate superconductor," Nature Materials, 12, 387-391 (2013)

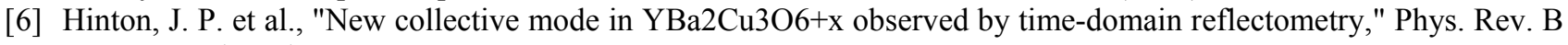
88,060508 (2013).

[7] Dakovski, G.L., Lee, W.-S., Hawthorn, D. G., Garner, N., Bonn, D, Hardy, W, Liang, R., Hoffmann, M.C. and Turner, J.J., "Enhanced coherent oscillations in the superconducting state of underdoped $\mathrm{YBa} 2 \mathrm{Cu} 3 \mathrm{O} 6+\mathrm{x}$ induced via ultrafast terahertz excitation," Phys. Rev. B, 91, 220506(R) (2015)

[8] Hebling, J., Almasi, G., Kozma, I. and Kuhl, J. "Velocity matching by pulse front tilting for large area THz-pulse generation," Optics Express, 10, 1161 (2002)

[9] Yeh, K.-L., Hoffmann, M. C. , Hebling, J. and Nelson, K.A., "Generation of $10 \mu \mathrm{J}$ ultrashort terahertz pulses by optical rectification," Appl. Phys. Lett., 90, 171121 (2007)

[10] Liang, R., Bonn, D. A. and Hardy, W. N., "Growth of high quality YBCO single crystals using BaZrO3 crucibles," Physica C 304, 105 (1998).

[11]Liang, R., Bonn, D. A. and Hardy, W. N., "Evaluation of $\mathrm{CuO} 2$ plane hole doping in $\mathrm{YBa} 2 \mathrm{Cu} 3 \mathrm{O} 6+\mathrm{x}$ single crystals," Phys. Rev. B 73, 180505 (2006).

[12] Saluzzo M. et al., "Thickness effect on the structure and superconductivity of Nd1.2Ba1.8Cu3Oz epitaxial films," Phys. Rev. B 72, 134521 (2005)

[13] Le Tacon, M. et al., "Inelastic X-ray scattering in $\mathrm{YBa}_{2} \mathrm{Cu}_{3} \mathrm{O}_{6}{ }_{6}$ reveals giant phonon anomalies and elastic central peak due to charge-density-wave formation," Nat. Phys. 10, 52-58 (2013).

[14] Larbalestier, D., Gurevich, A., Feldmann, D. M. and Polyanskii, "A. High-Tc superconducting materials for electric power applications," Nature 414, 368-377 (2001).

[15] Kabanov, V. V., Demsar, J., Podobnik, B. and Mihailovic, D., "Quasiparticle relaxation dynamics in superconductors with different gap structures: Theory and experiments on YBa2Cu3O7- $\delta$," Phys. Rev. B. 59, 1497 (1999). 